\title{
What Should We Prefer in the Surgical Management of Differentiated Thyroid Carcinomas?
}

\author{
Fatih Çiftçi \\ Istanbul Gelisim University, Vocational School of Healthy Services, Istanbul, Turkey
}

\section{ABSTRACT}

Aim: The study evaluates the results of surgical procedures performed in patients with differentiated thyroid carcinoma. There are many discussions regarding the optimal surgical treatment of differentiated thyroid carcinoma; their focal point is the type or extent of thyroidectomy.

Material and Method: We performed a retrospective analysis on 85 patients diagnosed with differentiated thyroid carcinoma, treated in the period July 2007 - April 2017. The average age the patients, consisting of 15 males and 70 females, was of 41 (17-85) years. The gross findings at operation and the prognostic factors such as patients' age, tumour size, local invasion, nodal involvement and the presence of distant metastasis were taken into account when deciding on the type of thyroidectomy, and cervical neck dissection.

Results: The most prominent symptom was the existence of a cervical mass (85\%); fineneedle aspiration biopsy (FNAB) was performed in 73 patients and revealed a diagnostic accuracy in 61 patients (72\%). Histological examination confirmed papillary carcinoma in 76 patients, follicular carcinoma in 8 patients and medullary carcinoma in 1 patient. When the diagnosis was established, 19 patients had palpable neck lymph nodes, and 25 patients had a local invasion to surrounding structures. While total thyroidectomy was performed in 71 $(83.5 \%)$ patients, 11 (12.4\%) patients underwent lobectomy and subtotal thyroidectomy, and $3(3.5 \%)$ patients underwent lobectomy and near-total lobectomy. Modified neck dissection was added to 13 patients who underwent total thyroidectomy. No operative mortality was observed, and the cancer-related mortality rate was $10.2 \%$. Temporary and permanent hypoparathyroidism rates were $5 \%$ and $1.1 \%$, respectively. Temporary and permanent recurrent nerve paralysis was observed to be of $3 \%$ and $0 \%$, respectively.

Conclusion: The selection of treatment for differentiated thyroid carcinoma should be made based on risk factors. All procedures such as lobectomy + subtotal lobectomy, near-total thyroidectomy, and total thyroidectomy are selected in safe conditions. In addition to total thyroidectomy, modified neck dissection should also be considered for patients with differentiated thyroid carcinoma with nodal involvement.

Key words: Differentiated thyroid carcinoma, total thyroidectomy, subtotal thyroidectomy, cervical lymph node dissection

\section{INTRODUCTION}

The ratio of thyroid carcinomas in all malignant tumours is $1 \%$, and their
Corresponding author:

Fatih Ciftci, MD

Basaksehir Mah., shakpașa sk, Bulvar stanbul sitesi, E4 Blok daire: 79Basaksehir, Istanbul, 34306

E-mail: oprdrfatihciftci@gmail.com 
incidence is reported to be 40 per million (1). Although four different histological types are defined, differentiated carcinomas constitute $85 \%$ of all thyroid cancers encountered (1). Certain risk factors, such as radiation history to the head and neck region, age, histology, tumour size, cervical nodal involvement and the presence of distant metastasis, are directly associated with the prognosis. For this purpose, the AMES scoring system was defined by Cady and Rossi and the AGES scoring system was described by Hay et al $(2,3)$. There is an ongoing discussion about the ideal surgical approach. While the fact to what extent thyroidectomy or with what type of thyroidectomy cure is achieved is discussed on the basis of patient selection and risk factors, it is argued that the possibility of local recurrence after total thyroidectomy will be substantially reduced, reoperative surgery will be eliminated and thus the feared complications after completion thyroidectomy can be prevented $(4,5,6)$. In patient follow-up, it was emphasized that serum thyroglobulin measurements would become more sensitive, and in the screening for recurrence or metastatic disease, increases would be achieved in ablation success with radioactive $\left.\right|^{131}$ in case of recurrence or metastatic disease $(7,8,9)$. On the other hand, discussions are also going on for nodal metastases in patients with differentiated thyroid carcinoma. In the past, while the high rate of nodal involvement directed thyroid surgeons to prophylactic neck dissections, the increasing trend is that local control in the neck can be achieved by therapeutic nodal dissections $(10,11)$.

This study aimed to perform an analysis of surgical procedures and other treatment modalities performed in patients with the diagnosis of differentiated thyroid carcinoma treated in our clinic.

\section{MATERIAL AND METHOD}

Eighty-five patients with differentiated thyroid carcinoma treated between July 2007 and April 2017 were included in the study. Thirty-two patients who underwent limited thyroidectomy and were later diagnosed with malignancy, including 23 patients with the diagnosis of solitary thyroid nodules in external centers and 9 patients in our clinic, were not included in the study. All patients in this group underwent total thyroidectomy, and their analyses are planned within the scope of a separate study when the documentation of clinicopathological data before and after the treatment is completed.

Furthermore, a patient who was understood to have parathyroid adenoma with differentiated thyroid carcinoma was not included in the study. The patients were divided into two different risk groups according to the AMES scoring system defined by Cady and Rossi (2) (table 1). The patients underwent medical investigations depending on their symptoms and physical findings, ultrasonographic and scintigraphic examinations, endocrinological studies and fine-needle aspiration cytology.

A transverse cervical incision was performed, and the incision was extended to the cervical upper lateral in patients with added neck dissection. Regardless of the type and extent of thyroidectomy, both recurrent nerves were identified in all patients, and at least two parathyroids were ensured to remain intact. The parathyroid glands which incorrectly remained in the specimen during the dissection of the thyroid were reimplanted into the sternocleidomastoid muscle, which is on the non-dominant side for the disease after thyroidectomy was completed. All surgical procedures were terminated by placing drains.

Unilateral or bilateral modified neck dissections were added to the patients whose cervical lymph node involvement was determined during the operation. Modified neck dissection was later performed in patients who were found to have nodal metastasis in the neck during the follow-up period. All postoperative

Table 1 - AMES criteria for the identification of risk groups in differentiated thyroid carcinomas

\begin{tabular}{l}
\hline Low-Risk Group \\
- All young patients without distant metastasis (males 41 years of age, females 51 years of age) \\
- Patients with intrathyroidal papillary cancer or follicular carcinoma with minor capsular invasion \\
- All elderly patients with primary cancer less than $5 \mathrm{~cm}$ and without distant metastasis \\
High-Risk Group \\
* All patients with distant metastasis \\
- Patients with extrathyroidal papillary cancer or follicular carcinoma with significant capsular involvement \\
- All elderly patients with primary cancer greater than $5 \mathrm{~cm}$ or regardless of the extent of the disease \\
\hline
\end{tabular}


patients underwent neck and total body scintigraphy at the end of the fourth week. When low $1^{131}$ uptake (<\%1) and elevated TSH( $>5 \mathrm{U} / \mathrm{ml}$ ) levels were reached, thyroxine hormone replacement was initiated. Furthermore, all patients underwent radioactive iodine ablation in the $30-100 \mathrm{mCi}$ dose range regardless of the type of thyroidectomy. Vocal cord examination was performed routinely in all patients in the preoperative and postoperative periods to evaluate the recurrent nerve paralysis. The patients were periodically controlled every 3 months for the first year, every 6 months for the second year and then once a year. In the neck examination after at least the 6-month diseasefree period for disease recurrence in the neck, the presence of palpable mass and elevated serum thyroglobulin values (> $3 \mathrm{ng} / \mathrm{mL}$ ) were identified according to the scintigraphic study and biopsy results. Two patients with local recurrence during the follow-up period after lobectomy + subtotal lobectomy underwent $\mathrm{I}^{131}$ ablation at an average dose of $115 \mathrm{mCi}$ (100-130 $\mathrm{mCi})$ in addition to thyroxine hormone replacement. Moreover, the presence of metastatic disease was detected by direct radiographic examinations and total body scintigraphy $\mathrm{I}^{131} . \mathrm{I}^{131}$ treatment at doses of 100-200 mCi for metastatic lung disease and 150-300 $\mathrm{mCi}$ for bone metastasis and limited local radiotherapy only for bone metastases were performed.

\section{RESULTS}

The patients constituting the series consisted of 15 males and 70 females, and their average age was 41 (17-85) years. Their histories stated that none of the patients underwent radiation for therapeutic purposes to the head and neck region in the pre-disease period. One patient had a history of familial goitre, and one patient had a history of endemic goitre. Except for head-neck examination, the systematic physical examinations of the patients were performed. It was determined that 19 patients (22\%) had dysphonia, 11 patients (12\%) had dysphagia, 72 patients $(85 \%)$ had cervical mass, and 24 patients (29\%) had cervical lymphadenopathy (LAP). While neck ultrasonography for thyroid and $\mathrm{Tc}^{99 "}$ scintigraphic study were performed in all patients, fine-needle aspiration biopsy (FNAB) was performed in 73 patients. FNAB identified a malignant lesion in 61 patients (72\%) and gave false negative values in 9 patients (16\%). The diagnosis was reported as a suspicious lesion in 3 patients (3.5\%) (table 2). While the diagnosis of differentiated thyroid carcinoma was obtained by frozen section biopsy in 27
Table 2 - Patient characteristics

\begin{tabular}{lcc}
\hline & Count(n) & $\%$ \\
\hline Patients number & 85 & \\
\hline Gender & 70 & 82.3 \\
$\quad$ Female & 15 & 17.6 \\
$\quad$ Male & 44 & \\
\hline Mean age & & \\
\hdashline Symptom & 19 & 22 \\
$\quad$ Dysphonia & 11 & 12 \\
Dysphagia & 72 & 85 \\
$\quad$ Mass of the neck & 24 & 29 \\
$\quad$ Lymphadenopathy of the neck & & \\
\hline Needle aspiration biopsy & 61 & 72 \\
$\quad$ Positive & 9 & 16 \\
$\quad$ Negative & 3 & 3.5 \\
Suspicious & 46 & 54.1 \\
\hdashline Low-risk group & 36 & \\
$\quad$ Papillary & 10 & \\
Follicular & 39 & 45.9 \\
High-risk group & 32 & \\
$\quad$ Papillary & 7 & \\
Follicular &
\end{tabular}

patients, the diagnosis of minimal thyroid carcinoma (tumour diameter $\leq 1 \mathrm{~cm}$ ) was reconfirmed in gross sections in 13 of them. A total of 85 patients with differentiated thyroid carcinoma were evaluated according to the AMES criteria, and it was observed that 46 patients $(54.1 \%)$ were in the low-risk group and 39 patients (45.9\%) were in the high-risk group (tables 1 and 2). The diagnosis was confirmed by frozen section biopsy in 12 patients with FNAB negative or suspicious lesion. While lobectomy + subtotal lobectomy was performed in 8 out of 10 patients with minimal thyroid carcinoma, 2 of them underwent lobectomy near-total lobectomy. No recurrent disease was observed within the follow-up period (table 2) in these patients who were followed up for an average of 61 months (15-93). While lobectomy + subtotal lobectomy was performed in 10 of the other 46 patients in the low-risk group, total thyroidectomy was performed in 36 of them. Of this group of patients with an average follow-up period of 44 months (19-66 months), local recurrence developed in two of them, who underwent lobectomy subtotal lobectomy. $1^{131}$ ablation was performed at an average dose of $115 \mathrm{mCi}$ (100-130 $\mathrm{mCi}$ in both patients showing resistance to completion thyroidectomy, and an apparent resolution was clinicoendocrinologically achieved. Neck scintigraphy revealed a minimal distribution of radioactivity in the local disease region along with a decrease in serum thyroglobulin levels. However, recurrence developed again in the old local recurrence disease region in one 
of these patients during the 8-month period following the treatment. Completion total thyroidectomy was performed for this patient who was convinced for secondary surgery. In this patient, no morbidity was observed other than temporary vocal cord paralysis in the post-operative period. While no cancer-related death was observed during the follow-up period, two patients died of myocardial infarction, and one patient died of trauma.

A total of 39 patients in the high-risk group underwent total thyroidectomy. In 32 of these patients, the tumour had a local invasion to adjacent soft structures through the thyroid capsule. In the neck examination, while the presence of unilateral LAP was found in 24 patients, it was also observed that 4 patients had contralateral LAP during the surgical exploration. In the patients included in the high-risk group, cervical lymph node involvement was investigated by frozen section biopsy from nodal specimens. Unilateral modified neck dissection was added to total thyroidectomy in 8 patients whose metastatic involvement in the same side lymph nodes was confirmed in the frozen section. It was found out that 2 patients had bilateral cervical lymph node involvement, and bilateral modified neck dissection was performed. Central compartment cleaning was performed in 7 patients whose lymph node was negative in frozen sections. However, ipsilateral cervical lymph node metastasis was detected in 2 patients during the follow-up period, and modified neck dissection was later performed in these patients. In 7 patients in the high-risk group, distant metastases occurred in the lungs (4 cases) and bones ( 3 cases) (tables 3 and 4). While metastasis from differentiated tumour to lung originated from papillary carcinoma in 4 patients and follicular carcinoma in 1 patient, metastasis to the skeletal system originated from papillary carcinoma in 1 patient and follicular carcinoma in 3 patients. While two bone metastases were detected in the skull, one of them was discovered in the left clavicle, and the other one was detected in the left humeral head. The mean duration of the occurrence of metastatic disease after surgery was found to be 25 months (6-66). The patients diagnosed with metastatic disease underwent radioactive iodine ablation and radiotherapy. Radioactive iodine treatment was administered at average doses of $147 \mathrm{mCi}$ (100-200 $\mathrm{mCi}$ ) for lung metastasis and $184 \mathrm{mCi}(150-300 \mathrm{mCi})$ for bone metastasis. Limited radiotherapy was performed in 4 patients for bone metastases.

While no operative mortality was observed in 85 operated patients with the diagnosis of differentiated thyroid carcinoma, cancer-related mortality was
Table 3 - Tumour characteristics

\begin{tabular}{lcc}
\hline & $\begin{array}{c}\text { Patients number } \\
(\mathbf{n}=\mathbf{8 5})\end{array}$ & $\%$ \\
\hline Tumour diameter (mean)cm & 2.2 & $(0.6-4.3)$ \\
\hline Hystologic type & 76 & \\
$\quad$ Papillary & 8 & 89.4 \\
$\quad$ Follicular & 1 & 9.4 \\
$\quad$ Medullary & 25 & 1.1 \\
\hdashline Local invasion & 13 & 29.4 \\
\hline Lymph node invasion & 11 & 15.2 \\
$\quad$ Same side & 2 & 12.9 \\
$\quad$ Opponent side & 2.3 \\
\hdashline Distant metastasis & 4 & \\
$\quad$ Lung & 3 & 4.7 \\
Bone & & 3.5 \\
\hline
\end{tabular}

observed only in 8 patients (10.2\%) with distant metastasis. As postoperative complications, while haemorrhage was observed in 4 patients (4.9\%), temporary hypoparathyroidism in 5 patients $(5 \%)$, permanent hypoparathyroidism in 1 patient $(0.5 \%)$ and temporary vocal cord paralysis in 3 patients (3.4\%) were observed, however, permanent vocal cord paralysis was not observed in any patient (table 5).

\section{DISCUSSION}

There is an ongoing discussion about the ideal surgical treatment of differentiated thyroid carcinomas.

The focal point of discussions is on what extent thyroidectomy and surgical methods ranging from lobectomy to total thyroidectomy are commonly used $(5,6)$. The belief that an extensive thyroidectomy that will not leave behind functional thyroid tissue will reduce the recurrence rates and provide convenience in postoperative follow-up and adjuvant treatment is the starting point for total thyroidectomy $(7,8.9)$. On the

Table 4 - Applied surgical methods

\begin{tabular}{lcc}
\hline Width of surgical intervention & $\begin{array}{c}\text { Patients number } \\
(\mathbf{n = 8 5})\end{array}$ & $\%$ \\
\hline Low-risk group & 46 & 54.1 \\
Lobectomy-subtotal lobectomy & 8 & 11.7 \\
Lobectomy-Near the total lobectomy & 2 & 2.3 \\
Total thyroidectomy & 36 & 42.3 \\
\hdashline High-risk group & 29 & 34.1 \\
Total thyroidectomy & 12 & 14.1 \\
Total thyroidectomy + & & \\
unilateralneck dissection & 8 & 9.4 \\
Total thyroidectomy + & & \\
Bilateral modified radical neck dissection & 2 & 2.3 \\
Total thyroidectomy + Cental neck dissection & 7 & 8.2 \\
\hline
\end{tabular}


Table 5 - Post-operative complications

Patients numbers $(\mathrm{n}=85)$

\begin{tabular}{lcc}
\hline Haemorragie & 6 & 7.0 \\
\hline Wound infection & 1 & 1.1 \\
\hline Temporary hypoparathyroidism & 4 & 5 \\
\hdashline Permanent hypoparathyroidism & 1 & 1.1 \\
\hdashline Temporary vocal cord paralysis & 3 & 3.5 \\
\hline Temporary vocal cord paralysis & 0 & 0 \\
\hline
\end{tabular}

other hand, total thyroidectomy is also advocated as a safe method against the high complication rates of curative secondary thyroidectomy $(12,13,14)$.

The history of radiation to the neck region, the possibility of a multicentric tumour, the presence of an ipsilateral tumour larger than $2 \mathrm{~cm}$, the findings in favour of a tumour on the opposite side, and cervical lymph node involvement were demonstrated to be risk factors requiring extensive surgical intervention for differentiated thyroid carcinomas $(15,16)$. In this study, a total of 85 patients with differentiated thyroid carcinoma were evaluated according to the AMES criteria (tables 1 and 2). In our series, when 13 (15.2\%) patients in the low-risk group were re-evaluated in paraffin sections, they were confirmed as minimal thyroid carcinoma. While lobectomy + subtotal lobectomy was performed in 11 of these patients, lobectomy + near-total lobectomy was performed in 2 of them (tables 3 and 4). No disease recurrence or distant metastasis was observed in these patients followed up for an average of 44 months. Although the follow-up period was not long, these results are similar to the literature experiences. While Vickery et al. (14) reported that they did not find recurrence in the ipsilateral thyroid bed and contralateral lobe after resection in minimal thyroid carcinomas that were later diagnosed as carcinoma, Hay reported $6 \%$ disease recurrence after 20-year follow-ups for equal-level thyroid carcinomas (15). Noguchi mentioned cancer recurrence in remnant tissue only in 6 out $(0.7 \%)$ of 867 patients with small thyroid carcinoma during their 20-year follow-up, preferred total thyroidectomy in 3 patients and partial thyroidectomy in all remaining patients and described total thyroidectomy as an excessive intervention regardless of palpable nodal disease (19). In the presence of a nodule with malignancy in the thyroid; we believe that lobectomy to the dominant side will be the curative treatment modality if the clinicopathologic evaluation indicates minimal thyroid carcinoma, and subtotal or near-total lobectomy will be the treatment modality if it indicates the other side. However, it should not be forgotten that there is a risk of developing cancer again at an advanced period of life compared to the healthy population, despite the presence of small tumours of the thyroid. Therefore, long-term follow-up is needed regardless of the extent of the treatment.

On the other hand, the presence of cervical lymph nodes without a palpable tumour in the thyroid may be the first finding in thyroid carcinomas. Park and Min reported that they made a diagnosis from lateral neck nodes in a group of patients with nonpalpable thyroid carcinoma, and they performed near-total lobectomy + cervical lymph node dissection in some of these patients and only hemithyroidectomy in some of them (20). Likewise, other authors, who believe that small carcinomas of the thyroid may be more aggressive than palpable tumours, turned to additional neck dissections along with total thyroidectomy for these tumours (21). Noguchi indicated that the radical neck dissection they performed in only 9 patients was quite an unnecessary intervention, and such an intervention could only be performed in the presence of nodal metastases defined in the contralateral deep cervical nodes (16). In our series, ipsilateral lobectomy + contralateral subtotal lobectomy was performed in 8 of 46 patients in the low-risk group, while total thyroidectomy was performed in 36 of them (table 4). In this group, while local recurrence occurred only in 2 patients who underwent ipsilateral lobectomy + subtotal lobectomy, no recurrence was observed in patients who underwent total thyroidectomy. ${ }^{131}$ ablation treatment with TSH suppression gave result in both patients with local recurrence in the residual thyroid tissue in the neck. However, the second recurrent disease that developed later in one of these patients required additional surgery, and the control of the disease was ensured by completion total thyroidectomy. The success achieved in similar local disease states with a radiosensitive appearance by radioactive iodine ablation may vary according to the size of the residual thyroid tissue. Secondary surgical intervention is an alternative approach in cases that do not respond to radioactive iodine treatment or if recurrent carcinoma has developed in the contralateral thyroid lobe after primary surgical resection.

In the practice of thyroid surgery, the tendency to completion total thyroidectomy is common, especially in patients who underwent partial thyroidectomy for the solitary thyroid nodule but were later diagnosed with differentiated thyroid carcinoma. The fact that the possibility of multicentric carcinoma increases the risk of subsequent local recurrence in the contralateral 
thyroid lobe and the expectation for a facilitating effect for ablation treatment when required after this approach is argued as the main indications for the method $(22,23)$. The incidence of residual carcinoma in the contralateral lobe obtained with completion thyroidectomy was reported to be $28 \%$ by some authors and $27 \%$ by Rao et al (20-21). Another author stated that the incidence of residual thyroid cancer in reoperation specimens was 31\%, and they emphasised that completion thyroidectomy was an appropriate option for lesions larger than $1 \mathrm{~cm}$ in the first operation because $0 \%$ of these patients had the potential for residual cancer (22). The reasons that cause surgeons to feel anxious about completion thyroidectomy are the difficulty of dissection to be caused by broken neck anatomy and the complications that may develop accordingly. The rates reported by Gulcelik et al (23) for permanent vocal cord paralysis and permanent hypoparathyroidism after completion thyroidectomy were $2.5 \%$. Park YM (24) mentioned only $2 \%$ temporary vocal cord paralysis in a series of 100 cases. We believe that secondary neck exploration and completion total thyroidectomy are appropriate options if residual thyroid tissue is more (greater than $2 \mathrm{~cm}$ ) in patients in whom limited thyroidectomy is performed, and recurrence occurs in the contralateral lobe. However, it should also be known that this procedure, which requires intensive care and experience, must be performed with the lowest morbidity in the centres with a high volume of patients. The ablation treatment performed with radioactive iodine in the early postoperative period depending on the extent of thyroidectomy may also improve treatment efficiency by destroying residual thyroid tissue. However, it should be kept in mind that the use of high and repeated doses of radioactive iodine may lead to significant systemic complications such as pulmonary fibrosis, temporary bone marrow suppression and leukaemia $(25,28)$. Lower recurrence and higher survival rates are reported in patients receiving radioactive iodine therapy after total thyroidectomy compared to those undergoing total thyroidectomy only, regardless of the extent of the disease and the tumor histology $(26,29)$. It is pointed out that if there is some thyroid tissue left after surgery, then $I^{131}$ administered will be primarily drawn by healthy thyroid tissue and thus it will be difficult for it to reach malignant cells which are the main target $(27,30)$. In another series, there were 900 recurrence and cancer mortalities after total thyroidectomy in low-risk carcinomas; on the other hand, 93 contralateral recurrences were mentioned after hemithyroidectomy + radioactive iodine treatment (30). When our results are evaluated together with the literature data, it can be said that lobectomy intervention to the dominant lesion side, contralateral subtotal or near-total lobectomy are not the only optimal method but have a therapeutic for differentiated thyroid carcinomas that are considered to be in the low-risk group, however, the tendency to total thyroidectomy in highly invasive tumours (such as highly invasive follicular carcinoma) is a more appropriate option in terms of treatment efficacy.

We aimed to reduce the possibility of recurrence while performing total thyroidectomy in all patients in the high-risk group and increasing treatment efficacy by remnant ablation when needed. Central lymph node dissections were added to patients without gross involvement while performing modified radical neck dissection in patients with gross nodal involvement by total thyroidectomy. Modified radical neck dissection was performed in 2 patients due to the cervical nodal invasion which developed after local control in the neck, except for 2 patients in this group. The reason for secondary neck exploration in both patients could be inadequate nodal sampling in the first operation and could also be considered as the subsequent development of existing micro-metastases as a result of the decreased immune response. It was reported that the presence of micrometastatic disease (occult nodal metastasis) in the neck reached up to $80 \%$ after prophylactic neck dissections performed in the past $(31,32)$. However, the incidence of recurrent disease was found to be low (13.5\%) after a 10-year follow-up without performing lymph node dissection $(31,32)$. Later, it was understood that the clinical significance in nodal involvement was observed in $7-8 \%$ of patients with differentiated thyroid carcinoma, and the tendency to more conservative interventions increased. The studies demonstrated that $92 \%$ of local control could be achieved in the neck by limited neck dissection (berry picking, etc.) in well-differentiated carcinomas with minimal nodal involvement (32). Probably, development stops and even regresses in such metastases along with the removal of the primary tumour. From this point of view, central lymph node dissection in addition to total thyroidectomy may be an appropriate option for patients whose nodal involvement cannot be demonstrated histologically. However, the development of nodal metastases in the neck in two of our patients indicated that more radical interventions such as MRBD could not be ignored in the presence of clinically palpable neck nodes without histological evidence in differentiated thyroid carcinomas. It was reported that nodal involvement 
was an independent variable for disease recurrence and survival especially in follicular tumours and negatively affected the prognosis $(32,33)$. While both patients who subsequently developed nodal metastases had follicular histology despite central compartment cleaning, these two patients underwent completion MRBD following the literature experiences. Based on the short follow-up results, central lymph node dissection should be considered according to operative findings in high-risk differentiated thyroid carcinomas without palpable nodular growth in the neck. We think that a planned MRBD without seeking for histological evidence is a more effective method against new metastases that may occur later in life if nodal involvement in the neck is clinically noticed.

While no operative mortality was observed in our patients, the cancer-related mortality rate was $10.2 \%$. Deaths occurred due to distant metastases in patients in the high-risk group. While the mean duration of clinical manifestation of metastatic disease was 25 months in patients who underwent MRBD or central neck dissection in addition to total thyroidectomy, TSH suppression and radioactive iodine treatment were inefficient in 7 patients, and radiotherapy was also ineffective in 4 of these patients. In two patients with lung metastasis, metastatic disease was controlled by radioactive iodine treatment. Pulmonary metastases in differentiated thyroid carcinomas usually can retain radioactive iodine. If the radioactive iodine uptake examined in these patients before the treatment is normal, it is known that radioactive iodine treatment provides prolonged survival and even cure (34-35). However, iodine uptake is poor for bone metastases, and aggressive radiotherapy can be tried as another option $(34,35)$.

In our series, failure to achieve the expected benefit in patients undergoing radioactive iodine treatment and radiotherapy (only for bone metastases) with the diagnosis of metastatic disease of differentiated thyroid carcinomas ( 3 lungs and 4 bones) requires aggressive surgical procedures for metastases in invasive distant metastases of differentiated thyroid carcinomas. However, there will be significant anatomical and functional losses in such interventions. The overall operative complications are summarized in table 5, temporary and permanent hypoparathyroidism in our series was observed by $22 \%$ and $4 \%$, respectively, and temporary and permanent recurrent nerve paralysis was observed by $4 \%$ and $1 \%$, respectively. These morbidity rates, which can be considered high for thyroid surgery, might be due to the type of thyroidectomy and additional neck dissections performed in the patients in our series.
Many authors consider the width of resection, further neck dissection, completion reoperation, and even patient volume of the thyroid surgeon as the risk factors for thyroidectomy morbidity $(36,37,38)$.

Based on the results of an extensive clinical trial, some authors emphasised that the rate of permanent hypoparathyroidism that increased to $4 \%$ after neck dissection and completion thyroidectomy along with total thyroidectomy did not increase after total thyroidectomy without lymph node dissection $(37,39)$. On the other hand, the rates reported for permanent recurrent nerve paralysis are between $0.5 \%$ and $1.8 \%$ $(37,39)$. No permanent nerve paralysis was observed in our series. Although the number of patients is small, the rate of permanent recurrent nerve paralysis "b1 can be considered optimal according to the literature data.

\section{CONCLUSION}

Definitive intervention can be planned without the need for frozen-section biopsy in patients with differentiated thyroid carcinoma with FNAB. However, total thyroidectomy or lobectomy + near-total lobectomy can be considered in suspicious lesions that could not be diagnosed as malignant tumour despite FNAB and then frozen section biopsy. Since the presence of palpable lymph nodes does not always indicate the presence of histopathological metastasis in patients diagnosed with differentiated thyroid carcinoma, nodal sampling is useful. Although negative histopathologic status in nodal sampling indicates a formal neck dissection as a new intervention, central lymph node dissection is a correct approach.

Conflict of Interest: None declared

Source and Support: Nil

\section{REFERENCES}

1. Cansu GB, Yılmaz N, Toru S, Sarı R, Gökhan Ocak G, Arıcı C, et al. Evaluation of Incidental Thyroid Nodules in Cancer Patients. J Natl Med Assoc. 2017;109(4):299-306.

2. Cady B, Rossi R. An expanded rewiev of risk group definition in differentiated thyroid carci- noma. Surgery. 1988;104:947-953.

3. Hay ID, Bergstralh ES, Goellner JR, et al: Predict- ing outcome in papillary thyroid carcinoma: developing a reliable prognostic scoring system ina cohort of 1779 patients surgically treated at one institution during 1940 through 1989. Surgery 1993;114:1050-1057.

4. Clark $\mathrm{OH}$ : Total thyroidectomy the treatment of choice for patients with differentiated thyroid cancer. Ann Surg 1982; 196:361-366.

5. Prpic M, Franceschi M, Romi M, Jukic T, Kusic Z. Thyroglobulin as a tumour marker in differentiated thyroid cancer-clinical considerations. Acta Clin Croat. 2018;57(3):518-527. 
6. Aashiq M, Silverman DA, Na'ara S, Takahashi H, Amit M. RadioiodineRefractory thyroid cancer: Molecular basis of redifferantion therapies, management, and novel therapies. Cancers(Basel).2019;17;(11):9-15.

7. Wong JB, Kaplan MM, Meyer KB, Pauker SG. Ablative radioactive iodine therapy for apparentiy localized thyroid carcinoma. A decision analytic perspective. Endocrinol Metab Clin North Am 1990;19:741-760.

8. Noguchi 5, Murakami N: The value of lymph-node dissection in patients with differentiated thyroid cancer. Surg Clin North Am 1987;67:251-256.

9. Noguchi S5, Murakami N, Yamashita H, et al Papillary thyroid carcinoma. Modified radical neck dissection improves prognosis. Arch Surg. 1988;133:276-280.

10. Mc Dow AD, Pitt SC. Extent of surgery for low-risk differentiated thyroid cancer. Surg Clin North Am. 2019;99(4):599-610.

11. Dejong SA, Demeter JG, Lawrence AM, Paloyan E: Necessity and safety of completion thyroidectomy for differentiated thyroid carcinoma. Surgery 1992;112:734-749.

12. Vaisman F, Tuttle RM. Clinical assessment and risk stratification in differentiated thyroid cancer. Endocrinol Metab Clin North Am. 2019; 48(1):99-108.

13. Han JM, Bae JC, Kim HI, Kwon S, Jeon MJ, Kim WG, et al. Clinica outcomes of differentiated thyroid cancer patients with Local recurrence or distant metastasis detected in old age. Endocrinol Metab(seoul). 2018;33(4):459-465.

14. Vickery AL, Wang CA, Walker AM. Treatment of intrathyroidal papillary carcinoma of the thyroid. Cancer 1990;60:2587-2595.

15. Hay, Grant CS, Bergstralh ES; et al. Unilateral total lobectomy; is it sufficient surgical treatment for patients with AMES low-risk papillary thyroid carcinoma? Surgery 1998; 124:928-966.

16. Noguchi S, Yamashita H, Murakami N, et al. Small carcinomas of the thyroid. A long-term follow up of 867 patients. Arch Surg 1996;131: 187-191.

17. Zanocco K. ASO Author Reflections: Preoperative risk stratification in differentiated thyroid cancer. Ann Surg Oncol. 2018;25(3): 878-879.

18. Mc Dow AD, Pitt SC. Extent of surgery for low-risk differentiated thyroid cancer. Surg Clin North Am. 2019;99(4):599-610.

19. M Dy B, Strajina V, Tuttle M, R Shaha A. Completion Thyrooidectomy: Revisited a Quarter of a Century Later. Ann Surg Oncol. 2019;26(2):694-696.

20. Wu CW, Sun H, Zhang G, Kim HY, Catalfamo A, Portinari M, et al. Staged thyroidectomy: A single Institution Perspective. Laryngoscope Investig Otolaryngol. 2018;3(4):326-332.

21. Riju J, Thomas S, Anila KR. Completion thyroidectomy in differentiated thyroid malignancy-A Prospective Analysis. Indian J Surg Oncol. 2019;10(1):130-134.

22. Nicholson KJ, Teng CY, McCoy KL, Carty SE, Yip L. Completion thyroidectomy: A risky undertaking? Am J Surg.2019;(19)30312-5.

23. Gulcelik MA, Dogan L, Akgul GG, Güven EH, Ersöz Gulcelik N. Completion thyroidectomy: Safer than thought. Oncol Res Treat. 2018;41(6):386-390.

24. Park YM, KIM JR, Oh KH, Cho JG, Baek SK, Kwon SY, et al. Comparison of functional outcomes after total thyroidectomy and completion thyroidectomy: Hypoparathyroidism and postoperative complications. Auris Nasus Larynx. 2019;46(1):101-105.

25. Valderrabono P, Khazai L, Thompson ZJ, Leon ME, Otto KJ, Hallanger-Johnson JE, et al. Impact of oncogene panel results on surgical management of cytologically indeterminate thyroid nodules. Head Neck. 2018;40(8):1812-1823.

26. Hirshoren N, Kaganov K, Weinberger JM, Glaser B, Uziely B, Mizrahi I, et al. Thyrodectomy practise after Implementation of the 2015 American thyroid Association Guidelines on Surgical options for patients with well-differentiated thyroid carcinoma. JAMA Otolaryngol Head Neck Surg. 2018;44(5):427-432.

27. Craig WL, Smart L, Fielding S, Ramsay C, Krukowski ZH. Long term outcomes of simple clinical risk stratification in management of differentiated thyroid cancer. Surgeon. 2018;16(5):283-291.

28. Tuttle RM, Zhang L, Shaha A. A Clinical framework to facilitate selection of patients with differentiated thyroid cancer for active surveillance or less aggressive initial surgical management. Expert Rev Endocrinol Metab. 2018; 13(2):77-85

29. Noguchi SS, Murakami N: The value of lymph-node dissection in patients with differentiated thyroid cancer. Surg Clin North Am. 1987;67:251-261.

30. Fernandez-Vanes L, Llorente JL, Garcia-abo P, Menendez M, Pedregal D, Rodrigo JP, et al. Management of differentiated thyroid carcinomas. Acta Otorrinolaringol Esp. 2019:70(4):200-206.

31. Chong ST, Tan KM, Kok CYL, Guan SP, Lai SH, Lim C, et al. IL13RA2 is differentially regulated in papillary thyroid carcinoma versus follicular thyroid carcinoma. J Clin Endocrinol Metab. 2019;45(3): 65-71

32. Albano D, Panarotto MB, Durmo R, Rodella C, Bertegna F, Giubbini $R$. Clinical and prognostic role of detection timing of distant metastases in patients with differentiated thyroid cancer. Endocrine. 2019;63(1):79-86.

33. Yu ST, Ge JN, Luo JY, Wei ZG, Sun BH, Lei ST. Treatment-related adverse effects with TKIs in patients with advanced or radioiodine refractory differentiated thyroid carcinoma: a systematic review and meta-analysis. Cancer Manag Res. 2019;14;11:1525-1532.

34. Khan SA, Khan MS, Arif M, Durr-e-Sasbih, Rahim MK, Ahmad I. Radiation Dose-rate Reduction Pattern in well-differentiated thyroid cancer treated with I-131. J Coll Physicians Surg Pak.2015;25(7): 510-3.

35. Gulcelik MA, Kuru B, Dincer H, Camlıbel M, Yuksel UM, Yenidoğan $\mathrm{E}$, et al. Complications of completion versus total thyroidectomy. Asian Pac J Cancer Prev. 2012;13(10):5225-8.

36. Avenia N, Monacelli M, Sanguinetti A, Santoprete S, Pecorielli R, Ragusa M, et al. Therapeutic options in locally advanced thyroid carcinoma. Our experience. Ann Ital Chir. 2012;83(6):481-5.

37. Doubleday A, Sippel RS. Surgical options for thyroid cancer and post-surgical management.2018;13(3):137-148.

38. Lombardi CP, De Waure C, Mariani M, Carnassale G, D'Amore A, Traini $E$, et al. Efficacy of continuous neuromonitoring in thyroid surgery: preliminary report of a single-center experience. Gland Surg. 2019;8(4):336-342.

39. Wagner $\mathrm{HE}$, Seiler $\mathrm{CH}$. Recurrent laryngeal nerve palsy after thyroid gland surgery. Br J Surg. 1994;81:226-228. 